\title{
A disintegrin and metalloproteinase with thrombospondin motif 1 (ADAMTS1) expression increases in acute aortic dissection
}

\author{
Yanxiang Gao ${ }^{1}$, Wenjing $\mathrm{Wu}^{1}$, Changan $\mathrm{Yu}^{2}$, Fangming Zhong ${ }^{1}$, Geng $\mathrm{Li}^{2}$, Wei Kong ${ }^{3}$ \\ \& Jingang Zheng ${ }^{1 *}$ \\ ${ }^{1}$ Department of Cardiology, China-Japan Friendship Hospital, Beijing 100029, China; \\ ${ }^{2}$ Central Laboratory of Cardiovascular Disease, China-Japan Friendship Hospital, Beijing 100029, China; \\ ${ }^{3}$ Key Laboratory of Molecular Cardiovascular Science, Ministry of Education, Department of Physiology and Pathophysiology, School of \\ Basic Medical Sciences, Peking University, Beijing 100191, China
}

Received August 19, 2015; accepted October 10, 2015; published online November 10, 2015

\begin{abstract}
Acute aortic dissection (AAD) is a life-threatening cardiovascular disease caused by progressive medial degeneration of the aortic wall. A disintegrin and metalloproteinase with thrombospondin motifs 1 (ADAMTS1) is a recently identified extracellular metalloproteinase participating in the development of vascular disease, such as atherosclerosis. In the present study, we found that ADAMTS1 was significantly elevated in blood samples from AAD patients compared with patients with acute myocardial infarction and healthy volunteers. Based on these findings, we established an AAD model by infusing angiotensin II in older mice. AAD was successfully developed in aorta tissues, with an incidence of $42 \%$ after 14 days in the angiotensin II group. Macrophage and neutrophil infiltration was observed in the media of the aorta, and ADAMTS1 overexpression was found in the aorta by Western blot and immunohistochemistry. Double immunofluorescence staining showed the expression of ADAMTS1 in macrophages and neutrophils. Consistent with the upregulation of ADAMTS1 in aortic dissection tissues, versican (a proteoglycan substrate of ADAMTS1) was degraded significantly more in these tissues than in control aortic tissues. These data suggest that the increased expression of ADAMTS1 protein in macrophages and neutrophils that infiltrated aortic tissues may promote the progression of AAD by degrading versican.
\end{abstract}

ADAMTS1, acute aortic dissection, angiotensin II, macrophage, neutrophil

Citation: Gao, Y., Wu, W., Yu, C., Zhong, F., Li, G., Kong, W., and Zheng, J. (2016). A disintegrin and metalloproteinase with thrombospondin motif 1 (ADAMTS1) expression increases in acute aortic dissection. Sci China Life Sci 59, 59-67. doi: 10.1007/s11427-015-4959-4

\section{INTRODUCTION}

Acute aortic dissection (AAD) is a potentially fatal disease of the adult aorta. The incidence of AAD in the general population has increased over the past 10 years, becoming a significant burden on healthcare systems (Sampson, 2014a, b). Although the sudden onset of severe sharp back or chest pain and computed tomography images are effective for diagnosis, no specific biomarkers are available for the

*Corresponding author (email: victorzheng@ sina.com) prompt diagnosis of AAD. Progressive medial degeneration of the aortic wall is known to be an important pathogenic factor for the development of AAD. However, the underlying mechanism that links medial degeneration and the onset of AAD remains unclear.

The recently identified metalloproteinase family ADAMTS (a disintegrin and metalloproteinase with thrombospondin motifs) has been implicated in the development and progression of many cardiovascular diseases (Jonsson-Rylander et al., 2005; Wagsater et al., 2008; Wang et al., 2009; Zhang et al., 2015). The family member a dis- 
integrin and metalloproteinase with thrombospondin motifs-1 (ADAMTS1) is a secreted protease that participates in various biological processes, such as ovulation, inflammation, and angiogenesis (Iruela-Arispe et al., 2003; Ng et al., 2006; Russell et al., 2003). ADAMTS1 functions in extracellular matrix (ECM) remodeling by digesting modular proteoglycans, such as versican, aggrecan, and brevican (Kuno et al., 2000; Russell et al., 2003). Furthermore, the increased expression of ADAMTS1 has been implicated in acute vascular events, such as atherosclerotic plaque rupture (Jonsson- Rylander et al., 2005; Sabatine et al., 2008). However, little is known about the involvement of ADAMTS1 in the development of AAD.

In the present study, we found that ADAMTS1 was significantly elevated in human blood samples from AAD patients. Based on these findings, we established a mouse model of AAD by infusing angiotensin II (Ang II) in older mice. The upregulation of ADAMTS1 expression in macrophages and neutrophils that infiltrated the aortic lesions was found in this model, which was associated with the increased degradation of versican. Altogether, our study provides the first evidence that ADAMTS1 release from macrophages and neutrophils might contribute to the development of AAD.

\section{RESULTS}

\section{Elevated levels of ADAMTS1 in blood samples from AAD patients}

We first screened the circulating levels of ADAMTS1 in blood samples from healthy control volunteers and patients with acute myocardial infarction (AMI) or AAD. No significant differences were observed in average age, the ratio of men to women, or the incidence of major risk factors among these groups (Table 1). As shown in Figure 1A, the AAD group exhibited significantly higher plasma levels of ADAMTS1 than the control or AMI group.
Table 1 Demographic and clinical characteristics of the patients and controls included in the study. The data are expressed as mean \pm SEM or the number (percentage) of subjects. AMI, acute myocardial infarction; AAD, acute aortic dissection.

\begin{tabular}{lccc}
\hline \multicolumn{1}{c}{ Characteristics } & $\begin{array}{c}\text { Control } \\
(n=19)\end{array}$ & $\begin{array}{c}\text { AMI } \\
(n=26)\end{array}$ & $\begin{array}{c}\text { AAD } \\
(n=16)\end{array}$ \\
\hline Age (years) & $58 \pm 2$ & $60 \pm 3$ & $55 \pm 4$ \\
Male sex, $n(\%)$ & $11(58 \%)$ & $20(77 \%)$ & $13(81 \%)$ \\
Smoking, $n(\%)$ & $10(53 \%)$ & $21(81 \%)$ & $9(56 \%)$ \\
Hypertension, $n(\%)$ & $12(63 \%)$ & $17(65 \%)$ & $11(69 \%)$ \\
Diabetes mellitus, $n(\%)$ & $4(21 \%)$ & $7(27 \%)$ & $1(6 \%)$ \\
Hyperlipidemia, $n(\%)$ & $5(26 \%)$ & $11(42 \%)$ & $3(19 \%)$ \\
\hline
\end{tabular}

\section{Immunolocalization of ADAMTS1 in aortic lesions in AAD patients}

Immunohistochemistry of the patient samples indicated that the AAD tissues contained abundant ADAMTS1-positive cells that were located mainly in the medial layer of dissected aorta (Figure 1B). Mononuclear and polymorphonuclear inflammatory cells accumulated in much higher levels in dissected aorta from AAD patients (Figure 1B). Because the ADAMTS1 staining pattern in the AAD aorta was similar to inflammatory cells, we performed double immunofluorescence staining of ADAMTS1+CD68 (macrophages) and ADAMTS1+neutrophil elastase (neutrophils) in AAD aortic tissues and found that both ADAMTS1+CD68 and ADAMTS1+neutrophil elastase colocalized in the cells (Figure 2).

\section{Establishment of an AAD model by Ang II infusion in older mice}

Based on the finding that ADAMTS1 is upregulated in human $\mathrm{AAD}$, we sought to define its role in $\mathrm{AAD}$ in vivo by developing a relevant mouse model. Ang II infusion was previously reported to induce aortic dissection (Tieu et al., 2009). We evaluated the effects of short-term Ang II infu-
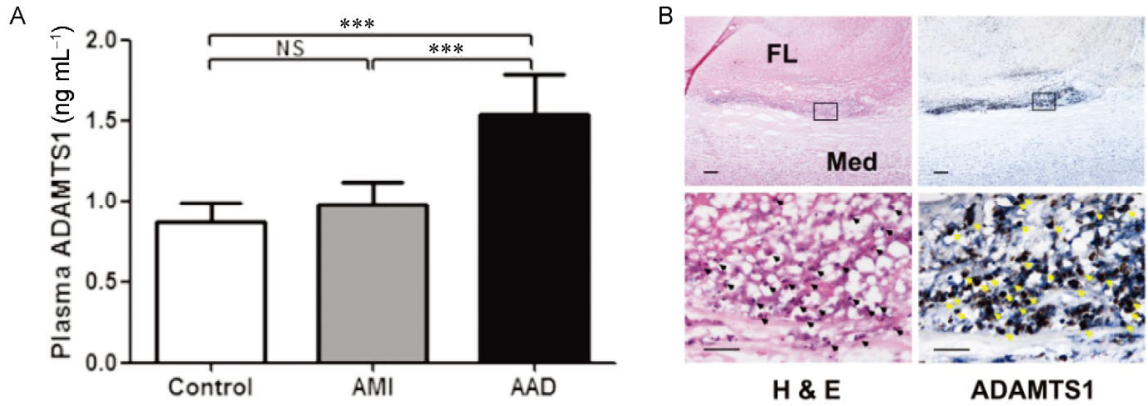

Figure 1 ADAMTS1 protein expression is elevated in blood and dissected aortic tissue samples from AAD patients, and dissected aortic media is infiltrated by numerous inflammatory cells. A, ADAMTS1 was assayed using an enzyme-linked immunosorbent assay for the marker in human peripheral blood samples from healthy volunteers $(n=19)$, AMI patients $(n=26)$, and AAD patients $(n=16)$. The data are expressed as mean \pm SEM. $* * *, P<0.001$. B, Aortic tissues were obtained from patients with AAD and subjected to histology and immunohistochemistry to detect ADAMTS1 in serial frozen sections. The distribution of ADAMTS1-positive cells and inflammatory cells was closely related. High magnification in the rectangular areas is shown in the lower rows in each panel. Black arrowheads indicate inflammatory cells. Yellow arrowheads indicate positively immunostained cells. Scale bar, 200 and $50 \mu m$ (upper and lower panels, respectively). Med, media; FL, false lumen; H\&E, hematoxylin and eosin stain. 


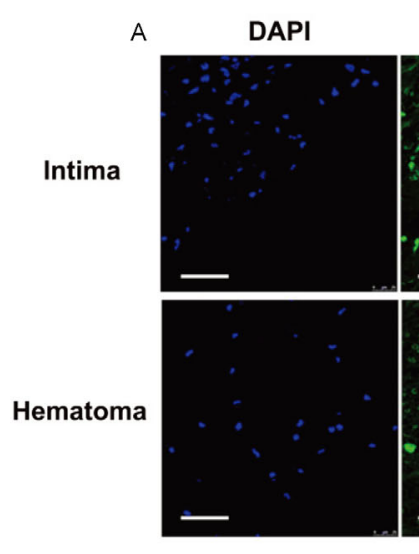

ADAMTS1 Neutrophil elastase
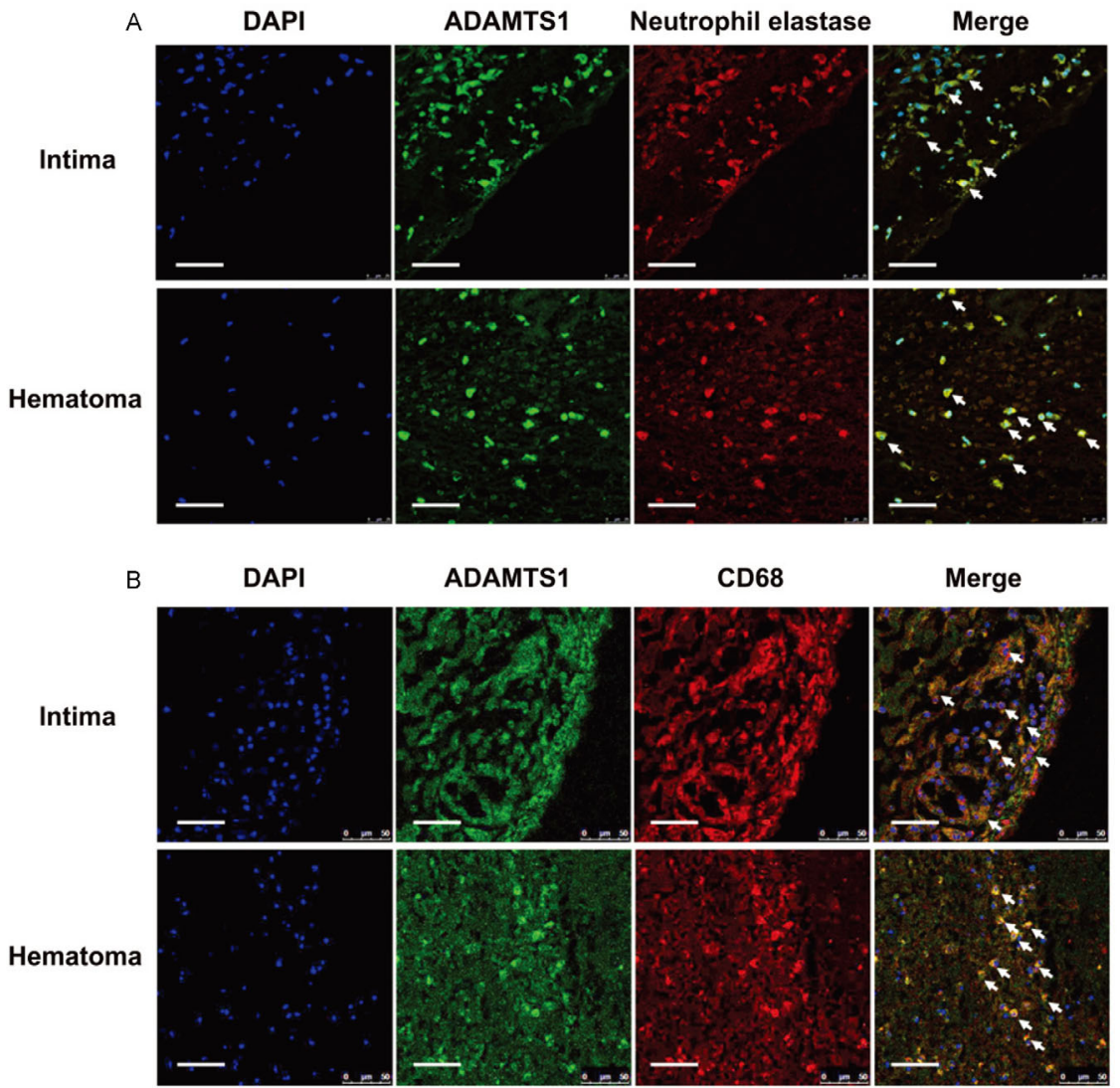

ADAMTS1
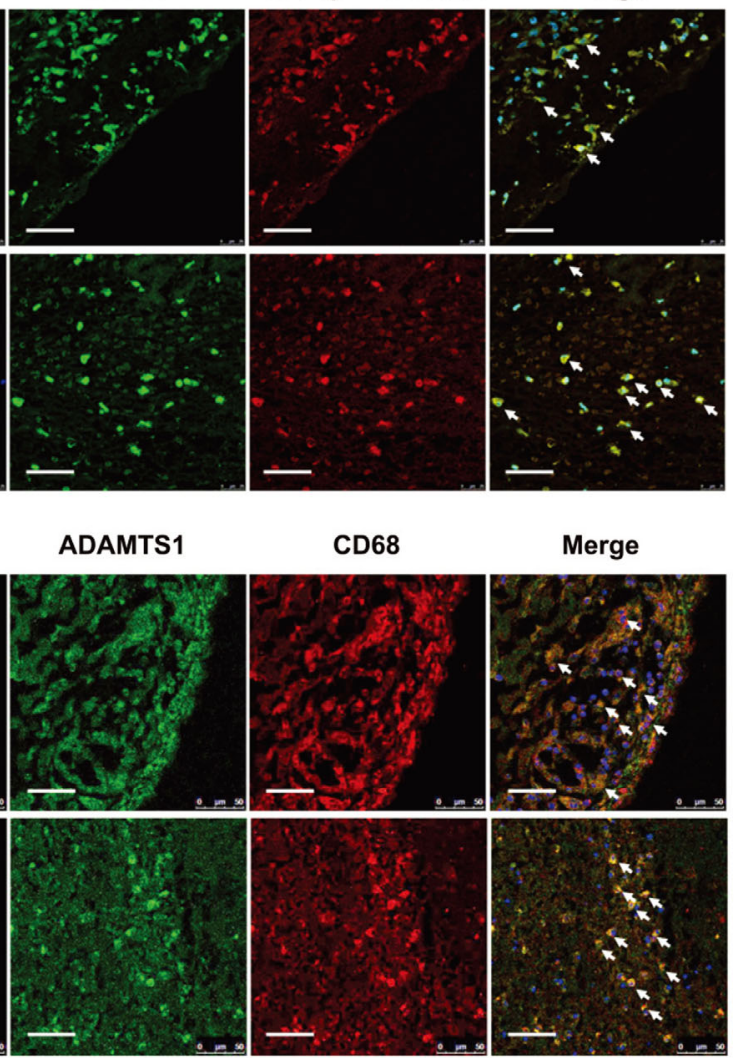

Figure 2 Dissected aortic media from AAD patients is infiltrated by numerous ADAMTS1-positive neutrophils and macrophages. A, Aortic tissues obtained from AAD patients were subjected to immunofluorescence staining for ADAMTS1 and neutrophil elastase (Neutrophil) on serial frozen sections. White arrows indicate ADAMTS1+neutrophil elastase-double-positive cells. B, Aortic tissues obtained from AAD patients were subjected to immunofluorescence staining for ADAMTS1 and CD68 (macrophage) on serial frozen sections. White arrows indicate ADAMTS1+CD68-double-positive cells. Scale bar, $50 \mu \mathrm{m}$.

sion in 8- to 9-month-old C57BL/6 mice at a dose of 2,500 $\mathrm{ng} \mathrm{kg}{ }^{-1} \min ^{-1}$. The infusion of Ang II at this dose produced modest pressure effects. Systolic blood pressure 7 days post-infusion was $154 \pm 5 \mathrm{mmHg}$ vs. $106 \pm 2 \mathrm{mmHg}$ preinfusion $(P<0.001$; Figure $3 \mathrm{~A})$. Aortic dissection was observed in $42 \%$ of the experimental mice (5 of 12) after intraventricular phosphate-buffered saline perfusion to remove lumenal blood cells (Figure 3B). Aortic dissection was not observed in sham-infused control mice. Furthermore, $80 \%$ of the mice suffering from aortic dissection (4 of 5 ) died as a result of aortic rupture. Dissections commonly spanned the length of the aorta from the thoracic to suprarenal aorta (Figure 3C). The analysis of aortic cross-sections in the suprarenal abdominal region clearly showed Ang II-induced medial hypertrophy, adventitial thickening, and blood-filled false lumens located in the tunica adventitia (Figure 3D).

\section{Elevated plasma ADAMTS1 levels in early stage of Ang II-induced AAD in older mice}

We detected the circulating levels of ADAMTS1 and Creactive protein (CRP) in blood samples from control and
Ang II-infused mice at day 3, 7 and 14 after replacement of the minipumps. As shown in Figure 4, the Ang II-treated group exhibited significantly higher plasma levels of ADAMTS1 and CRP than the control group at day 3 and 7. In addition, the plasma ADAMTS 1 levels were elevated more significantly, and the increased plasma CRP levels lasted longer. No significant differences were observed in plasma ADAMTS1 levels between two groups at day 14 after implantation of the minipumps.

\section{Immunolocalization of ADAMTS1 in aortic lesions in Ang II-infused mice}

Histology showed that the accumulation of inflammatory cells was localized to the media of the dissected aorta in Ang II-treated mice (Figure 3D). We performed double immunofluorescence staining of ADAMTS1+CD68 and ADAMTS1+neutrophil elastase in AAD aortic tissues and found that ADAMTS1+CD68 and ADAMTS1+neutrophil elastase colocalized in the cells (Figure 5). More importantly, the infiltration of ADAMTS1-positive macrophages and neutrophils was observed not only in the dissected media but also in the intima and adventitia in Ang II-induced AAD 
A

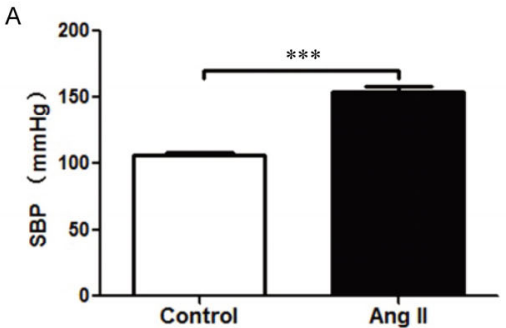

B

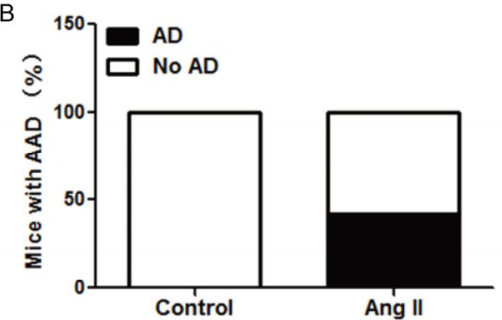

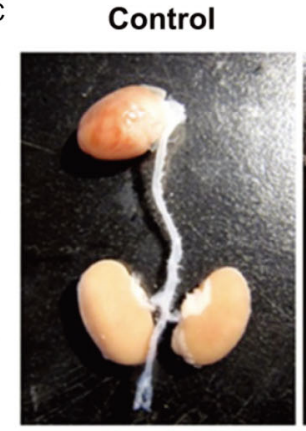

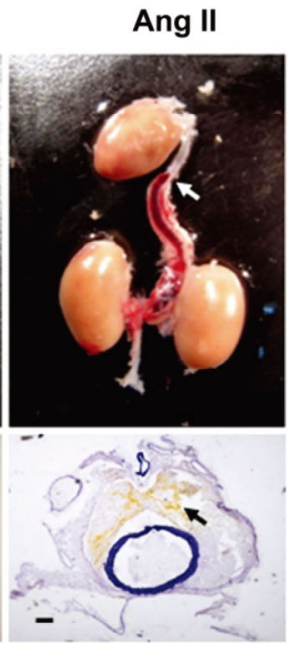

D

Control
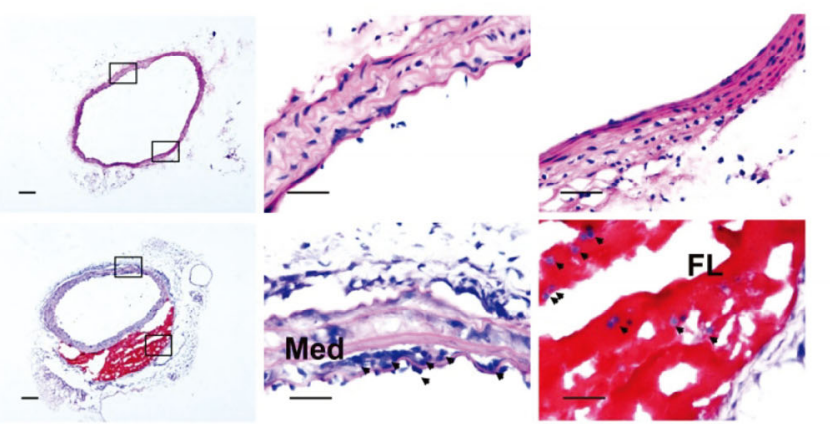

Ang II
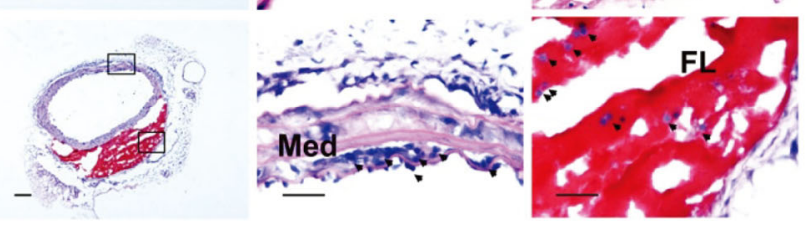

Figure 3 Angiotensin II infusion in older mice induces AAD. A, Systolic blood pressure was measured in saline- and Ang II-treated older mice ( $n=12$ per group). $* * *, P<0.001$. B, Incidence of AAD in older mice infused with saline or Ang II. C, Representative excised aortas show that older mice that were infused with Ang II developed AAD (upper). The figure shows the histology of aortas from control and Ang II-treated mice stained with elastica Van Gieson (lower). Notice the aortic dissection (arrowheads, false lumen) in the Ang II-treated mouse. Scale bar, $200 \mu \mathrm{m}$. D, Dissected aortic media from AAD mice is infiltrated by numerous inflammatory cells. Aortic tissues were obtained from control and Ang II-treated mice and subjected to histology using H\&E. High magnification of the rectangular areas is shown in the right panels in each row. Black arrowheads indicate inflammatory cells. Scale bar, $200 \mathrm{~m}$ and $50 \mu \mathrm{m}$ (left and right panels, respectively). SBP, systolic blood pressure; Med, media; FL, false lumen; H\&E, hematoxylin and eosin stain.

thoracic aortas (Figure 5). In control mice, the immunofluorescence staining of macrophages or neutrophils revealed hardly any ADAMTS1 expression in the tissues (Figure 5). These findings suggest that the increased levels of ADAMTS1 released from macrophages and neutrophils in AAD aortas might be involved in the development of AAD.

\section{Increased ADAMTS1 expression and versican degrada- tion in aortic tissues in Ang II-infused mice}

We examined the mRNA levels of ADAMTS1, the ADAMTS1 inhibitor tissue inhibitor metalloproteinases 3 (TIMP3), and the ADAMTS1 substrate versican using reverse-transcription PCR (RT-PCR) in mouse aortic tissues 14 days after Ang II infusion. The elevated mRNA expression of TIMP3 and versican was observed in aortas in Ang II-treated mice (Figure 6A-C). Western blot showed that the levels of ADAMTS1 and the versican degradation product DPEAEE were significantly higher and the level of versican was significantly lower in AAD tissues than in control aortic tissues (Figure 6D-G). The levels of versican degradation products strongly correlated with the levels of ADAMTS1 protein, suggesting a crucial role for ADAMTS1 in the degradation of versican in AAD.

\section{DISCUSSION}

In the present study, we found that the plasma levels of ADAMTS1 were elevated in AAD patients but not in patients with AMI or healthy control volunteers. Furthermore, increased circulating levels of ADAMTS1 were correlated with the presence of ADAMTS1-positive macrophages and neutrophils accumulated in aortic tissues in AAD patients. Given these findings from studies of human AAD specimens, we established a mouse model that develops AAD by Ang II infusion. Consistent with findings in AAD patients, plasma ADAMTS1 levels in Ang II-induced AAD mice were markedly elevated. Aortic tissue from AAD mice exhibited enhanced expression of ADAMTS1, and ADAMTS1-immunoreactive macrophages and neutrophils infiltrated dissected intima, media, and adventitia. Moreo- 

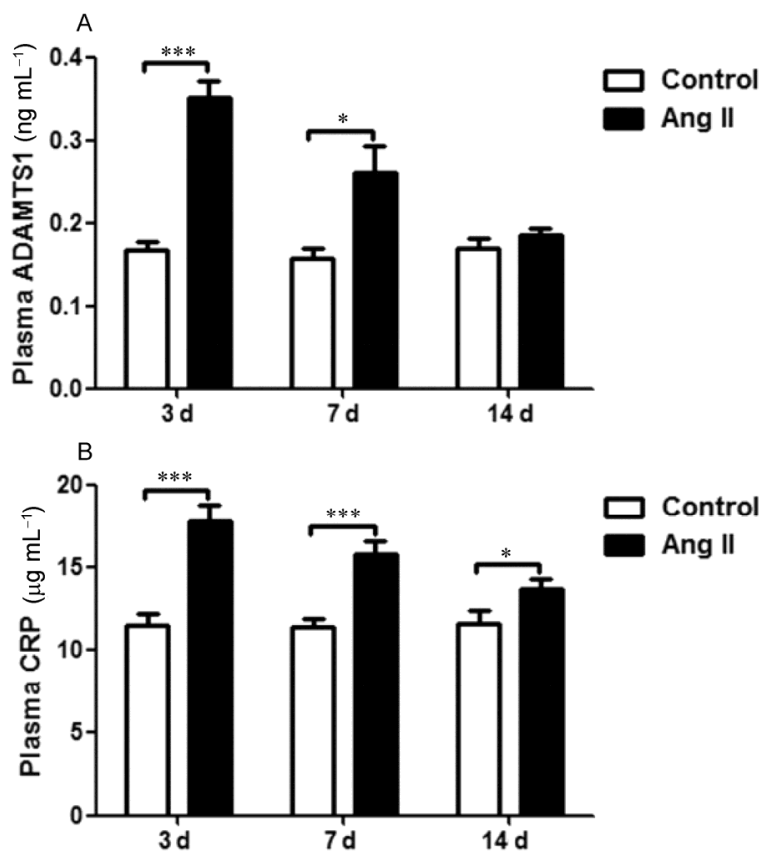

Figure 4 Circulating levels of ADAMTS1 and CRP are elevated in blood samples from Ang II-treated older mice. ADAMTS1 (A) and CRP (B) were assayed using enzyme-linked immunosorbent assays for the markers in mouse peripheral blood samples from older mice treated with vehicle (control) or Ang II. The data are expressed as mean \pm SEM. *, $P<0.05$, ***, $P<0.001$.

ver, the levels of ADAMTS1 protein were strongly correlated with the levels of versican degradation in dissected tissues. Our results suggest that ADAMTS1 may play an important role in the development of AAD.

Despite the technical advances in and resources dedicated to the treatment of $\mathrm{AAD}$, it is a potentially fatal vascular disease with high mortality. Early detection of AAD is clearly needed. AAD is pathologically associated with degeneration of aortic media, which is characterized by smooth muscle cells loss along with the degradation of extracellular matrix (Wu et al., 2013). Conversely, AMI is characterized by acute cardiomyocyte necrosis due to myocardial ischemia, triggering an intense inflammatory response (Fang et al., 2015). Our results suggest that ADAMTS1 may contribute to the loss of structural and functional integrity of the aortic wall in the progression of AAD by the degradation of versican, an extracellular matrix protein. This might explain that the circulating levels of ADAMTS1 were elevated only in AAD but not in AMI patients in our study, albeit both conditions are highly inflammatory. ADAMTS1 may therefore serve as a biomarker of differential diagnosis for AAD and AMI.

Matrix metalloproteinases are candidates in the pathological molecular mechanisms of AAD (Zhang et al., 2009). These enzymes degrade the ECM, modulate inflammation, and regulate tissue remodeling. Kurihara et al. (2012) demonstrated that MMP9 was elevated in the pathogenesis of aortic dissection, and the genetic deletion or pharmaceu- tical inhibition of MMP9 attenuated the incidence of AAD. Similarly, we observed that MMP9 protein expression was increased in aortic tissues in Ang II-treated mice in the present study (Figure S1).

ADAMTS is a recently identified family of extracellular metalloproteinases that has been shown to participate in tissue destruction (Kuno et al., 1997). The ADAMTS family of proteins comprises 20 members. ADAMTS1 is a particularly well-studied member of this family. Accumulating evidence suggests that ADAMT1 plays an important role in cardiovascular disease (Ashlin et al., 2013; JonssonRylander et al., 2005; Seidelmann et al., 2008). Jonsson-Rylander et al. showed that ADAMTS1 promoted atherogenesis by cleaving ECM proteins, such as versican, and promoting vascular smooth muscle cell migration (JonssonRylander et al., 2005). Consistent with our findings, Ren et al. also found that ADAMTS1 and ADAMTS4 expression was significantly increased in the aortic wall of human thoracic aortic aneurysm and dissection (Ren et al., 2013). However, they enrolled patients with chronic aortic dissection, and the time interval since dissection onset to test was several years. But we enrolled patients with acute aortic dissection, and the blood and tissue samples from AAD patients were collected within hours after dissection onset. Furthermore, they only found ADAMTS1 expression was increased in the aortic wall of human thoracic aortic aneurysm and dissection, but we proved that ADAMTS1 expression was similarly elevated in plasma and aortic tissues from patients with acute aortic dissection and Ang II-induced mouse models. In addition, they showed ADAMTS1 expression in smooth muscle cells and macrophages of dissected tissues, but we observed ADAMTS1 expression in neutrophils and macrophages accumulated in aortic tissues, but not in smooth muscle cells. Although there were many differences in detail between the findings in the present study versus those reported by Ren et al, these two studies suggest that ADAMTS1 played an important role in the different stages of aortic dissection.

We believe that ADAMTS1-knockout or knockdown animals may better reveal the role of ADAMTS1 in the pathogenesis of aortic dissection. If the occurrence of aortic dissection is inhibited in ADAMTS1-knockout or knockdown mice infused with Ang II, it would greatly strengthen our suppositions that ADAMTS1 might promote the development of aortic dissection. However, the targeted disruption of the ADAMTS1 gene resulted in ovarian defects associated with severely impaired fertility (Boerboom et al., 2003). In addition, it was senseless to inhibit ADAMTS1 expression in vivo in our study by tail-vein injection of specific siRNA adenovirus, since the elevated expression of ADAMTS1 mainly located in macrophages and neutrophils of dissected tissue, and leucocytes cannot be infected with adenovirus due to lack of adenovirus-specific receptors. At present, we could not get a sufficient number of ADAMTS1-knockout or knockdown mice to demonstrate 


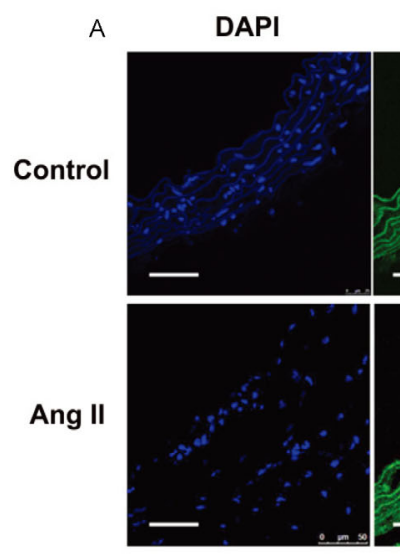

ADAMTS1 Neutrophil elastase

Merge
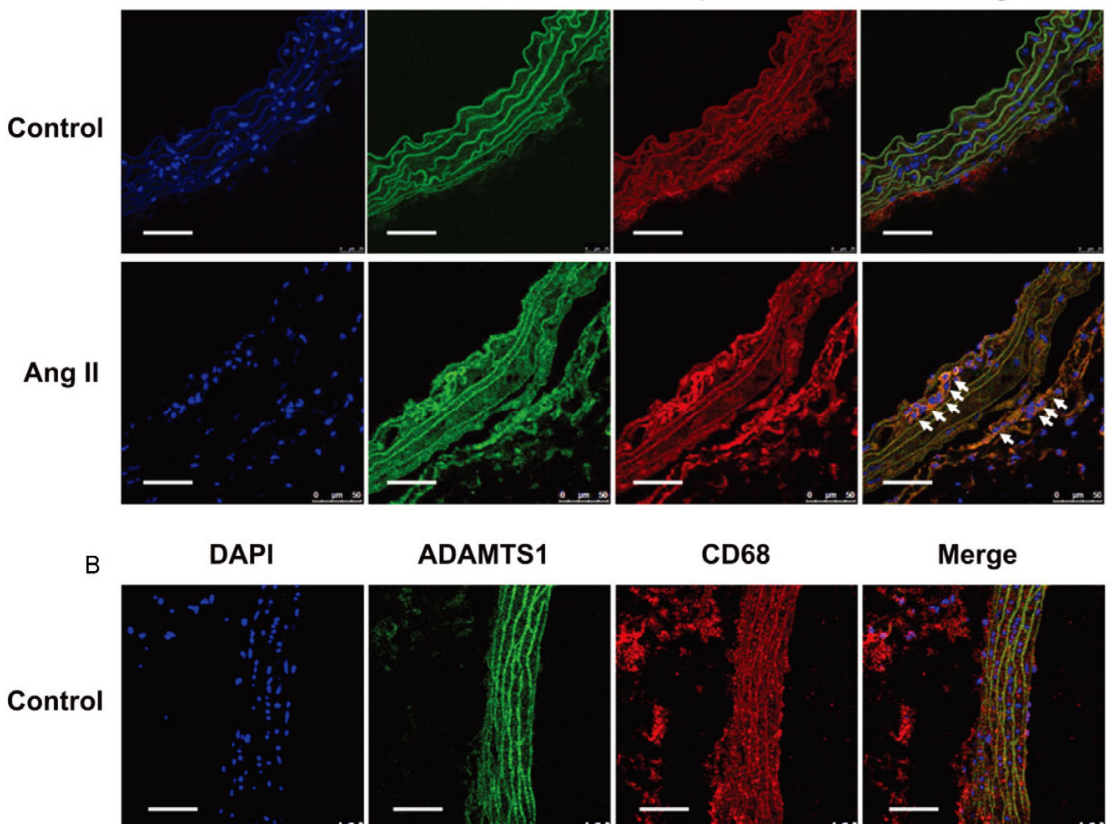

ADAMTS1

CD68

Merge
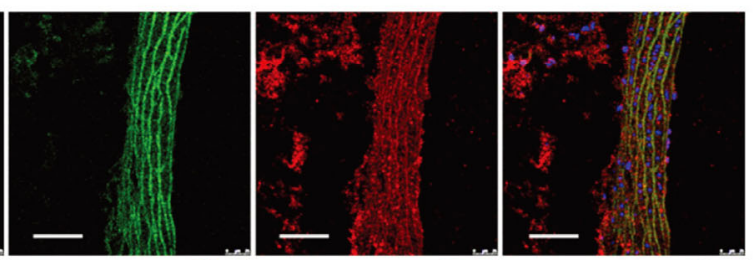

Ang II
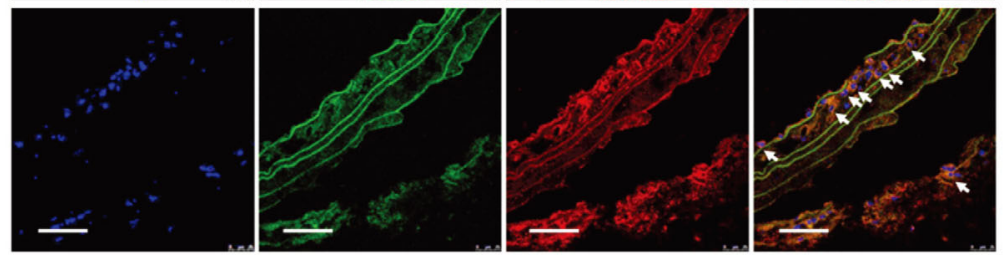

Figure 5 Dissected aortic media from AAD mice were infiltrated by numerous ADAMTS1-positive neutrophils and macrophages. A, Aortic tissues were obtained from the control and Ang II-treated mice and subjected to immunofluorescence staining for ADAMTS1 and neutrophil elastase (Neutrophil) on serial frozen sections. White arrows indicate ADAMTS1+neutrophil elastase-double-positive cells. B, Aortic tissues were obtained from the control and Ang II-treated mice and subjected to immunofluorescence staining for ADAMTS1 and CD68 (macrophage) on serial frozen sections. White arrows indicate ADAMTS1+CD68-double-positive cells. Scale bar, $50 \mu \mathrm{m}$.

that the increased expression of ADAMTS1 was required for the development of aortic dissection.

Versican is a major proteoglycan component of the vascular wall. It is mainly expressed in smooth muscle cells and can be cleaved by various enzymes, including ADAMTS1 (Jonsson-Rylander et al., 2005). Versican exists in at least four different isoforms, including $\mathrm{V}_{0}, \mathrm{~V}_{1}, \mathrm{~V}_{2}$, and $\mathrm{V}_{3}$, which are created by the alternative splicing of mRNA from a single gene (Wight and Merrilees, 2004). Although studies suggest that versican may be involved in vascular smooth muscle cell migration, proliferation, and lowdensity lipoprotein oxidation processes, such as retention, and participate in the development of atherosclerosis and other vascular diseases. The role of versican in vascular physiology and pathophysiology has not yet been clarified. A genetic analysis of familial thoracic aortic aneurysms and aortic dissection patients found that chromosome 5q13-14 is a highly pathogenic site for the disease (Guo et al., 2001), and the versican gene is located at this locus. The concentration of versican in the arterial wall was $89 \%$ lower in patients with aneurysms (Theocharis et al., 2001). Further research found a significant increase in the versican degra- dation fragment in the vascular wall in aortic aneurysm tissues (Theocharis et al., 2003). These studies suggest that versican degradation may be associated with the progression of aortic dissection or aneurysm. In the present study, we found that versican degradation increased in aortic tissues in Ang II-treated mice, accompanied by an increase in ADAMTS1 protein expression. The increased mRNA levels of versican and TIMP3 might reflect feedback changes associated with versican degradation and elevated ADAMTS1 expression. This strong correlation suggests that the elevation of ADAMTS1 protein levels may be responsible for the increase in versican degradation.

In summary, ADAMTS1 levels were elevated in patients with AAD and may contribute to the progression of AAD. ADAMTS1 could serve as a potential biomarker for AAD and possible therapeutic target for the treatment of AAD.

\section{MATERIALS AND METHODS}

\section{Human blood and affected aortic samples}

Between January 2011 and December 2013, 16 patients 


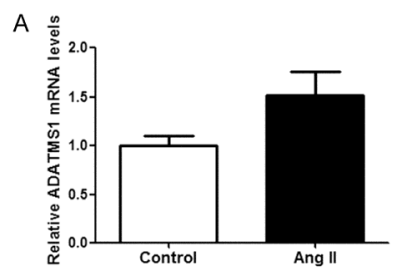

B

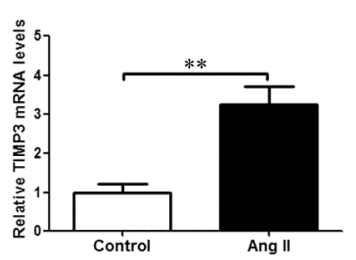

D Control Ang II

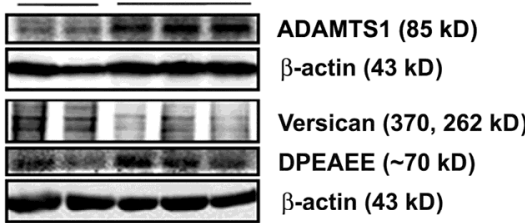

$\mathrm{E}$

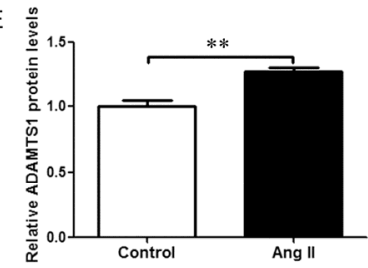

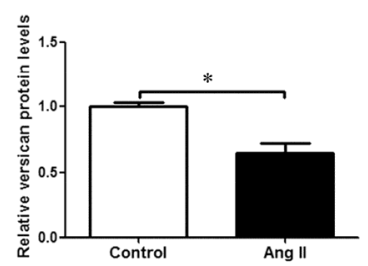

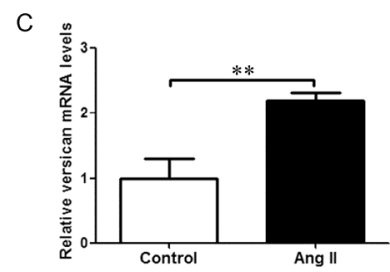

G

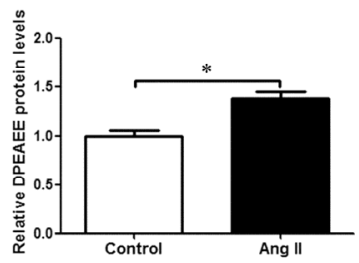

Figure 6 Acute aortic dissection in Ang II-treated older mice is accompanied by enhanced ADAMTS1 expression and versican degradation. Analysis of ADAMTS1 (A), TIMP3 (B), and versican (C) mRNA expression by real-time polymerase chain reaction in aortic tissues from older mice treated with vehicle (control) or Ang II. Polymerase chain reaction products from three independent samples from each group are shown. D, Representative Western blots of ADAMTS1, versican, and the degradation product of versican (DPEAEE) in aortic tissues from older mice treated with vehicle (control) or Ang II. Analysis of ADAMTS1 (E), versican (F), and DPEAEE $(\mathrm{G})$ protein expression by Western blot. The data are expressed as mean \pm SEM. $*, P<0.05 ; * *, P<0.01$. TIMP3, tissue inhibitor of metalloproteinases3.

who were diagnosed with AAD, 26 patients with AMI, and 19 healthy volunteers were enrolled in the study. All of the AAD patients were free from connective tissue disorders, such as Marfan syndrome, Ehlers-Danlos syndrome, and aortitis diagnosed according to clinical history and physical examination. The blood samples from AAD and AMI patients were collected within $1 \mathrm{~h}$ after arrival in the hospital's emergency room. AAD and AMI patients who arrived at the hospital $10 \mathrm{~h}$ after the onset of clinical symptoms were excluded from the study. The diagnosis of AAD and AMI was confirmed by computed tomography and electrocardiogram, respectively. The concentrations of ADAMTS1 in blood samples were analyzed using a human ADAMTS1 enzyme-linked immunosorbent assay (ELISA) kit (MyBioSoure, San Diego, CA, USA). Affected aortic specimens were obtained at surgery from 25 AAD patients, who underwent surgery for aortic grafts. The human samples were collected in the China-Japan Friendship Hospital, and written informed consent for the usage of the samples for the experiments was obtained from all of the subjects. The study was approved by the ethics committee of China-Japan Friendship Hospital.

\section{Animal care and use}

Eight- to nine-month-old male C57BL/6 mice were purchased from the Animal Center of Peking University Health Science Center (Beijing, China) and maintained in a specific pathogen-free facility at the Experimental Center of China-
Japan Friendship Hospital (accredited for animal care by the Chinese Association for Accreditation of Laboratory Animal Care) under a $12 \mathrm{~h} / 12 \mathrm{~h}$ light/dark cycle with controlled room temperature and free access to standard chow and tap water. Two groups of mice ( $n=12$ per group) received continuous Ang II (2,500 $\mathrm{ng} \mathrm{kg}^{-1} \mathrm{~min}^{-1}$; Sigma Chemical, St. Louis, MO, USA) or saline via subcutaneous osmotic minipumps (Alza, Palo Alto, CA, USA) for 14 days. We take the blood of mice at day 3, 7 and 14 after implantation of the minipumps. The concentrations of ADAMTS1 and CRP in blood samples were analyzed using a mouse ADAMTS1 ELISA kit (MyBioSoure, San Diego, CA, USA) and a mouse C-reactive protein ELISA kit (ADI, San Antonio, TX, USA).

\section{Blood pressure measurement}

Arterial systolic blood pressure (SBP) and heart rate (HR) were measured in conscious, restrained mice using a noninvasive computerized tail-cuff system (Muromachi Kikai, Tokyo, Japan). Measurements were performed every day, beginning 2 days before placement of the minipumps. The blood pressure value for each mouse was calculated as the average of three separate measurements in each session.

\section{Histology and immunohistochemistry}

Paraformaldehyde-fixed tissue was embedded with optimum cutting temperature compound or paraffin and pro- 
cessed, and $6 \mu \mathrm{m}$ sections were stained with hematoxylin-eosin or elastica Van Gieson. Additional sections were immunostained using an indirect horseradish peroxidase immunoperoxidase method and specific antibody for the antigen of ADAMTS1 (Abcam, Cambridge, MA, USA). Negative controls for the immunostaining consisted of replacing each of the primary antibodies with an equivalent concentration of irrelevant rabbit polyclonal antibody. At least three different specimens were analyzed.

\section{Immunofluorescence staining}

For the dual immunofluorescence assay, frozen sections were incubated with anti-ADAMTS1 (Abcam, Cambridge, MA, USA), anti-CD68, or anti-neutrophil elastase (Santa Cruz, CA, USA) primary antibody and then Alexa Fluor 488 donkey anti-rabbit immunoglobulin $\mathrm{G}(\mathrm{IgG})$ or Alexa Fluor 555 donkey anti-goat IgG (Invitrogen, Carlsbad, CA, USA) secondary antibody. Nuclei were counterstained with 4',6-diamidino-2-phenylindole. Negative controls replaced primary antibody with $\mathrm{IgG}$. The fluorescence signal was monitored by confocal laser scanning microscopy (Leica, Wetzlar, Germany).

\section{Quantitative real-time RT-PCR}

Total RNA from mouse aortas was isolated using Trizol reagent (Applygen Technologies, Beijing, China) and reverse-transcribed with a reverse transcription system (Promega, Madison, WI, USA). The reaction mixture then underwent polymerase chain reaction (PCR). The amount of PCR products formed in each cycle was evaluated by SYBR Green I fluorescence. Amplification reactions were performed using the ABI Prism 7500 PCR System. All of the amplification reactions underwent 35 cycles and were performed in duplicate (an initial stage of $5 \mathrm{~min}$ at $95^{\circ} \mathrm{C}$, followed by a three-step cycle of $30 \mathrm{~s}$ at $95^{\circ} \mathrm{C}, 30 \mathrm{~s}$ at $60^{\circ} \mathrm{C}$, and $30 \mathrm{~s}$ at $72^{\circ} \mathrm{C}$ ). The accuracy of the PCR products was confirmed by sequencing the amplicons. The relative target mRNA levels were normalized to the internal control, $\beta$-actin. The following primers were used: ADAMTS1 (forward, TTTGGCCAGCAGCTACATCT; reverse, CACACCTTCACAGAGGCTGA), TIMP3 (forward, CACGGAAGCCTCTGAAAGTC; reverse, AATTGCAACCCAGGTGGTAG), versican (forward, CAAGCCAAAATGGAAACCAG; reverse, CCCCTTGTAGTCCTGACCAA), $\beta$-actin (forward, ATCTGGCACCACACCTTC; reverse, AGCCAGGTCCAGACGCA).

\section{Western blot}

The extracts of mouse tissue were collected using tissue lysis buffer (Beyotime, Jiangsu, China) plus $1 \mathrm{mmol} \mathrm{L}^{-1}$ phenylmethylsulfonyl fluoride. Total protein was quantified using a bicinchoninic acid protein assay (Pierce, Rockford, IL, USA). Proteins were separated by $10 \%$ sodium dodecyl sulfate-polyacrylamide gel electrophoresis and electropho- retically transferred to nitrocellulose membranes, which were incubated with specific antibodies for the antigens of $\beta$-actin (MBL, Nagoya, Japan), ADAMTS1 (Abcam, Cambridge, MA, USA), versican (Millipore, Temecula, CA, USA), and DPEAEE (LifeSpan BioSciences, Seattle, WA, USA), washed, and incubated with an appropriate IRDye800-conjugated second antibody (Rockland, Gilbertsville, PA, USA). Specific immunofluorescence bands were detected using the Odyssey infrared imaging system (LI-COR Biosciences, Lincoln, NE, USA).

\section{Statistical analysis}

All of the results are expressed as the mean \pm SEM or original data that represented one of at least three independent experiments. The data were analyzed using an unpaired Student's $t$-test and two-way analysis of variance with Prism software (GraphPad, La Jolla, CA, USA) followed by the Student-Newman-Keuls post hoc test. $P<0.05$ was considered statistically significant.

Compliance and ethics The author(s) declare that they have no conflict of interest. This study was performed in strict accordance with the recommendations of the Guide for the Care and Use of Laboratory Animals of the National Institutes of Health. The protocol was approved by the Committee on the Ethics of Animal Experiments of China-Japan Friendship Hospital. All of the surgeries were performed in sodium pentobarbital-anesthetized mice, and all efforts were made to minimize suffering.

Acknowledgements This work was supported by the National Natural Science Foundation of China (81170287).

Ashlin, T.G., Kwan, A.P., and Ramji, D.P. (2013). Regulation of ADAMTS-1, -4 and -5 expression in human macrophages: Differential regulation by key cytokines implicated in atherosclerosis and novel synergism between TL1A and IL-17. Cytokine 64, 234-242.

Boerboom, D., Russell, D.L., Richards, J.S., and Sirois, J. (2003). Regulation of transcripts encoding ADAMTS-1 (a disintegrin and metalloproteinase with thrombospondin-like motifs-1) and progesterone receptor by human chorionic gonadotropin in equine preovulatory follicles. $\mathbf{J}$ Mol Endocrinol 31, 473-485.

Fang, L., Moore, X.L., Dart, A.M., and Wang, L.M. (2015). Systemic inflammatory response following acute myocardial infarction. J Geriatr Cardiol 12, 305-312.

Guo, D., Hasham, S., Kuang, S.Q., Vaughan, C.J., Boerwinkle, E., Chen, H., Abuelo, D., Dietz, H.C., Basson, C.T., Shete, S.S., and Milewicz, D.M. (2001). Familial thoracic aortic aneurysms and dissections: genetic heterogeneity with a major locus mapping to 5q13-14. Circulation 103, 2461-2468.

Iruela-Arispe, M.L., Carpizo, D., and Luque, A. (2003). ADAMTS1: a matrix metalloprotease with angioinhibitory properties. Ann N Y Acad Sci 995, 183-190.

Jonsson-Rylander, A.C., Nilsson, T., Fritsche-Danielson, R., Hammarstrom, A., Behrendt, M., Andersson, J.O., Lindgren, K., Andersson, A.K., Wallbrandt, P., Rosengren, B., Brodin, P., Thelin, A., Westin, A., Hurt-Camejo, E., and Lee-Sogaard, C.H. (2005). Role of ADAMTS-1 in atherosclerosis: remodeling of carotid artery, immunohistochemistry, and proteolysis of versican. Arterioscler Thromb Vasc Biol 25, 180-185.

Kuno, K., Kanada, N., Nakashima, E., Fujiki, F., Ichimura, F., and Matsushima, K. (1997). Molecular cloning of a gene encoding a new type of metalloproteinase-disintegrin family protein with thrombospondin mo- 
tifs as an inflammation associated gene. J Biol Chem 272, 556-562.

Kuno, K., Okada, Y., Kawashima, H., Nakamura, H., Miyasaka, M., Ohno, H., and Matsushima, K. (2000). ADAMTS-1 cleaves a cartilage proteoglycan, aggrecan. FEBS Lett 478, 241-245.

Kurihara, T., Shimizu-Hirota, R., Shimoda, M., Adachi, T., Shimizu, H., Weiss, S.J., Itoh, H., Hori, S., Aikawa, N., and Okada, Y. (2012). Neutrophil-derived matrix metalloproteinase 9 triggers acute aortic dissection. Circulation 126, 3070-3080.

Ng, Y.H., Zhu, H., Pallen, C.J., Leung, P.C., and MacCalman, C.D. (2006). Differential effects of interleukin-1beta and transforming growth factor-beta1 on the expression of the inflammation-associated protein, ADAMTS-1, in human decidual stromal cells in vitro. Hum Reprod 21, 1990-1999.

Ren, P., Zhang, L., Xu, G., Palmero, L.C., Albini, P.T., Coselli, J.S., Shen, Y.H., and LeMaire, S.A. (2013). ADAMTS-1 and ADAMTS-4 levels are elevated in thoracic aortic aneurysms and dissections. Ann Thorac Surg 95, 570-577.

Russell, D.L., Doyle, K.M., Ochsner, S.A., Sandy, J.D., and Richards, J.S. (2003). Processing and localization of ADAMTS-1 and proteolytic cleavage of versican during cumulus matrix expansion and ovulation. $\mathrm{J}$ Biol Chem 278, 42330-42339.

Sabatine, M.S., Ploughman, L., Simonsen, K.L., Iakoubova, O.A., Kirchgessner, T.G., Ranade, K., Tsuchihashi, Z., Zerba, K.E., Long, D.U., Tong, C.H., Packard, C.J., Pfeffer, M.A., Devlin, J.J., Shepherd, J., Campos, H., Sacks, F.M., and Braunwald, E. (2008). Association between ADAMTS1 matrix metalloproteinase gene variation, coronary heart disease, and benefit of statin therapy. Arterioscler Thromb Vasc Biol 28, 562-567.

Sampson, U.K., Norman, P.E., Fowkes, F.G., Aboyans, V., Song, Y., Harrell, F.E., Forouzanfar, M.H., Naghavi, M., Denenberg, J.O., McDermott, M.M., Criqui, M.H., Mensah, G.A., Ezzati, M., and Murray, C. (2014a). Estimation of global and regional incidence and prevalence of abdominal aortic aneurysms 1990 to 2010. Glob Heart 8, 159-170.

Sampson, U.K., Norman, P.E., Fowkes, F.G., Aboyans, V., Song, Y., Harrell, F.E., Forouzanfar, M.H., Naghavi, M., Denenberg, J.O., McDermott, M.M., Criqui, M.H., Mensah, G.A., Ezzati, M., and Murray, C. (2014b). Global and regional burden of aortic dissection and aneurysms. Glob Heart 8, 171-180.
Seidelmann, S.B., Kuo, C., Pleskac, N., Molina, J., Sayers, S., Li, R., Zhou, J., Johnson, P., Braun, K., Chan, C., Teupser, D., Breslow, J.L., Wight, T.N., Tall, A.R., and Welch, C.L. (2008). Athsq1 is an atherosclerosis modifier locus with dramatic effects on lesion area and prominent accumulation of versican. Arterioscler Thromb Vasc Biol 28, 2180-2186.

Theocharis, A.D., Tsolakis, I., Hjerpe, A., and Karamanos, N.K. (2001). Human abdominal aortic aneurysm is characterized by decreased versican concentration and specific downregulation of versican isoform $\mathrm{V}(0)$. Atherosclerosis 154, 367-376.

Theocharis, A.D., Tsolakis, I., Hjerpe, A., and Karamanos, N.K. (2003). Versican undergoes specific alterations in the fine molecular structure and organization in human aneurysmal abdominal aortas. Biomed Chromatogr 17, 411-416.

Tieu, B.C., Lee, C., Sun, H., Lejeune, W., Recinos, A., 3rd, Ju, X., Spratt, H., Guo, D.C., Milewicz, D., Tilton, R.G., and Brasier, A.R. (2009). An adventitial IL-6/MCP1 amplification loop accelerates macrophage-mediated vascular inflammation leading to aortic dissection in mice. J Clin Invest 119, 3637-3651.

Wagsater, D., Bjork, H., Zhu, C., Bjorkegren, J., Valen, G., Hamsten, A., and Eriksson, P. (2008). ADAMTS-4 and -8 are inflammatory regulated enzymes expressed in macrophage-rich areas of human atherosclerotic plaques. Atherosclerosis 196, 514-522.

Wang, L., Zheng, J., Bai, X., Liu, B., Liu, C.J., Xu, Q., Zhu, Y., Wang, N., Kong, W., and Wang, X. (2009). ADAMTS-7 mediates vascular smooth muscle cell migration and neointima formation in balloon-injured rat arteries. Circ Res 104, 688-698.

Wight, T.N., and Merrilees, M.J. (2004). Proteoglycans in atherosclerosis and restenosis: key roles for versican. Circ Res 94, 1158-1167.

Wu, D., Shen, Y.H., Russell, L., Coselli, J.S., and LeMaire, S.A. (2013). Molecular mechanisms of thoracic aortic dissection. J Surq Res 184, 907-924.

Zhang, L., Yu, F., Wang, L., Zheng, J., Du, Y., Huang, Y., Liu, B., Wang, X., and Kong, W. (2015). ADAMTS-7 promotes vascular smooth muscle cells proliferation in vitro and in vivo. Sci China Life Sci 58, 674-681

Zhang, X., Shen, Y.H., and LeMaire, S.A. (2009). Thoracic aortic dissection: are matrix metalloproteinases involved? Vascular 17, 147-157.

Open Access This article is distributed under the terms of the Creative Commons Attribution License which permits any use, distribution, and reproduction in any medium, provided the original author(s) and source are credited.

\section{SUPPORTING INFORMATION}

Figure S1 Representative Western blots of MMP9 in aortic tissues from older mice treated with vehicle (control) or Ang II. MMP9, matrix metalloproteinase 9; Ang II, angiotensin II.

The supporting information is available online at life.scichina.com and link.springer.com. The supporting materials are published as submitted, without typesetting or editing. The responsibility for scientific accuracy and content remains entirely with the authors. 